AUTHOR(S): B. G. Strait, H. R. Dye, and K. E. Kroncke

SUBMITTED TO 1991 IEEE Nuclear Science Symposium and Medical Imaging Conference Santa Fe, New Mexico

November 2-9, 1991

(Full Paper)

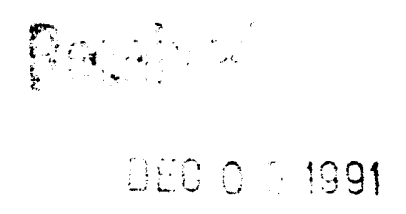

TITLE: A NEW, LOW-PROFILE NEUTRON DETECTOR JUNCTION BOX

\begin{abstract}
DISCLAIMER
This report was prepared as an account of work sponsored by an agency of the United States Government. Neither the United States Government nor any agency thereof, nor any of their employees, makes any warranty, express or implied, or assumes any legal liability or responsibility for the accuracy, completeness, or usefulness of any information, apparatus, product, or process disclosed, or represents that its use would not infringe privately owned rights. Reference herein to any specific commercial product, process, or service by trade name, trademark, manufacturer, or otherwise does not necessarily constitute or imply its endorsement, recommendation, or favoring by the United States Government or any agency thereof. The views and opinions of authors expressed herein do not necessarily state or reflect those of the United States Government or any agency thereof.
\end{abstract}




\title{
A NEW, LOW-PROFILE NEUTRON DETECTOR JUNCTION BOX*
}

\author{
B. G. Strait, H. R. Dye, and K. E. Kroncke \\ Safeguards Assay Group, N-1 \\ Los Alamos National Laboratory \\ Los Alamos, New Mexico
}

\section{ABSTRACT}

We have designed a new junction box for nondestructive assay instruments that is easier to build, maintain, and repair. Most high-voltage components are sealed from the atmosphere using potting material, therefore eliminating the maintenance requirement of replacing desiccators. The mechanical design cuts the cost of machining and makes it easy to remove and replace the ${ }^{3} \mathrm{He}$ tubes.

Neutron detectors in nondestructive assay instruments use junction boxes to distribute the high voltage to the ${ }^{3} \mathrm{He}$ tubes, to house the amplifier circuits, and to hold the ${ }^{3} \mathrm{He}$ tubes mechanically. The current design of a junction box is shown in Fig. 1. Operationally, this design has proven satisfactory although it does have several deficiencies, principally that it requires maintenance of desiccators to remove moisture from the high-voltage cavity of the junction box. As the desiccators become saturated with moisture, they must be replaced. Moisture in the high-voltage cavity causes high-voltage leakage or breakdown, which generates counts that are not related to neutrons. The new junction box design uses a potting material to seal out moisture from the high-voltage cavity and thus eliminates the maintenance problem of changing desiccators.

The current junction box design is 3-in. tall and 4-in. deep. The new design has a profile of only 1.5 in. and is 3.125 in. deep allowing 0.875 in. of polyethylene to be placed between the junction box and the instrument cavity if needed.

High-voltage distribution to the ${ }^{3} \mathrm{He}$ tubes in the conventional junction box is made by plugging a small-pin connector into the connector for the tube high-voltage interface for the ${ }^{3} \mathrm{He}$ tubes and soldering a bus wire between the tubes to this small-pin connector. This wiring procedure requires the tubes to be screwed in place in the junction box during the fabrication and remain in place during the junction box assembly. The problem with this design is that if the ${ }^{3} \mathrm{He}$ tubes need to be removed for replacement or removed because of detector space constraints during installation, the small-pin connectors

*This work was sponsored by the US Deparment of Energy, Office of Safeguards and Security. must be removed from each tube and replaced during the reassembly. The high-voltage cavity of the conventional junction box is located below the amplifier cavity. To disassemble the high-voltage circuits, as just described, one must also disassemble the amplifier circuits to obtain access to the highvoltage cavity. Significant disassembly and rewiring are necessary to remove tubes from the conventional junction box. Simple removal and replacement of ${ }^{3} \mathrm{He}$ tubes was a design goal for the new junction box, as discussed later.

Manufacturing cost is always a concern in producing detectors. The new junction box design simplifies the mechanical fabrication of the junction box and reduces the wiring cost. The new junction box is less costly to build. It is machined from standard 1.5 in. by 1.5 in. aluminum stock with only simple drilling and milling. The adjacent junction boxes and the input connectors to the first amplifier card are connected with flat cables on which the connectors are easily installed.

Figure 2 is a drawing of the new low-profile junction box. Parts ( $A$ and $C$ ) of the drawing show the four cavities that the tubes screw into and the two cavities that contain the input/output printed circuit cards. The cross-sectional view of part (D) shows the insert for the interface with the ${ }^{3} \mathrm{He}$-tube connector. It is nnachined out of KEL-F material, which provides high-voltage insulation between the high voltage on the pin and the junction box case. Bias voltage, usually about $1680 \mathrm{Vdc}$, is distributed to the four tubes using a bus wire soldered to the top of the four pins. A slot is machined in the ends of the pins to accommodate the bus wire. The KEL-F insert is press fitted into the cavity screw threads and epoxied to the junction box case at the top interface of the insert. The pin is also epoxied into the hole of the insert. The KEL-F insert and pin arrangement provides an interface that allows easy insertion and removal of the ${ }^{3} \mathrm{He}$ tube into and out of the junction box without having to rewire the junction box.

The ${ }^{3} \mathrm{He}$ tube output signal is a small current pulse produced as a result of neutrons interacting with the ${ }^{3} \mathrm{He}$ gas. The signal is coupled to an Amptek amplifier (see below for description) through a coupling capacitor. The coupling capacitor and a bleed resistor are mounted on a small-printed-circuit card and placed in the output cavity with an electrical connection to the bus wire. The output signal is transmitted to the Amptek amplifier by a small coax cable, RG174. Figure 3, par (B), is a schematic diagram of the output circuit. The 

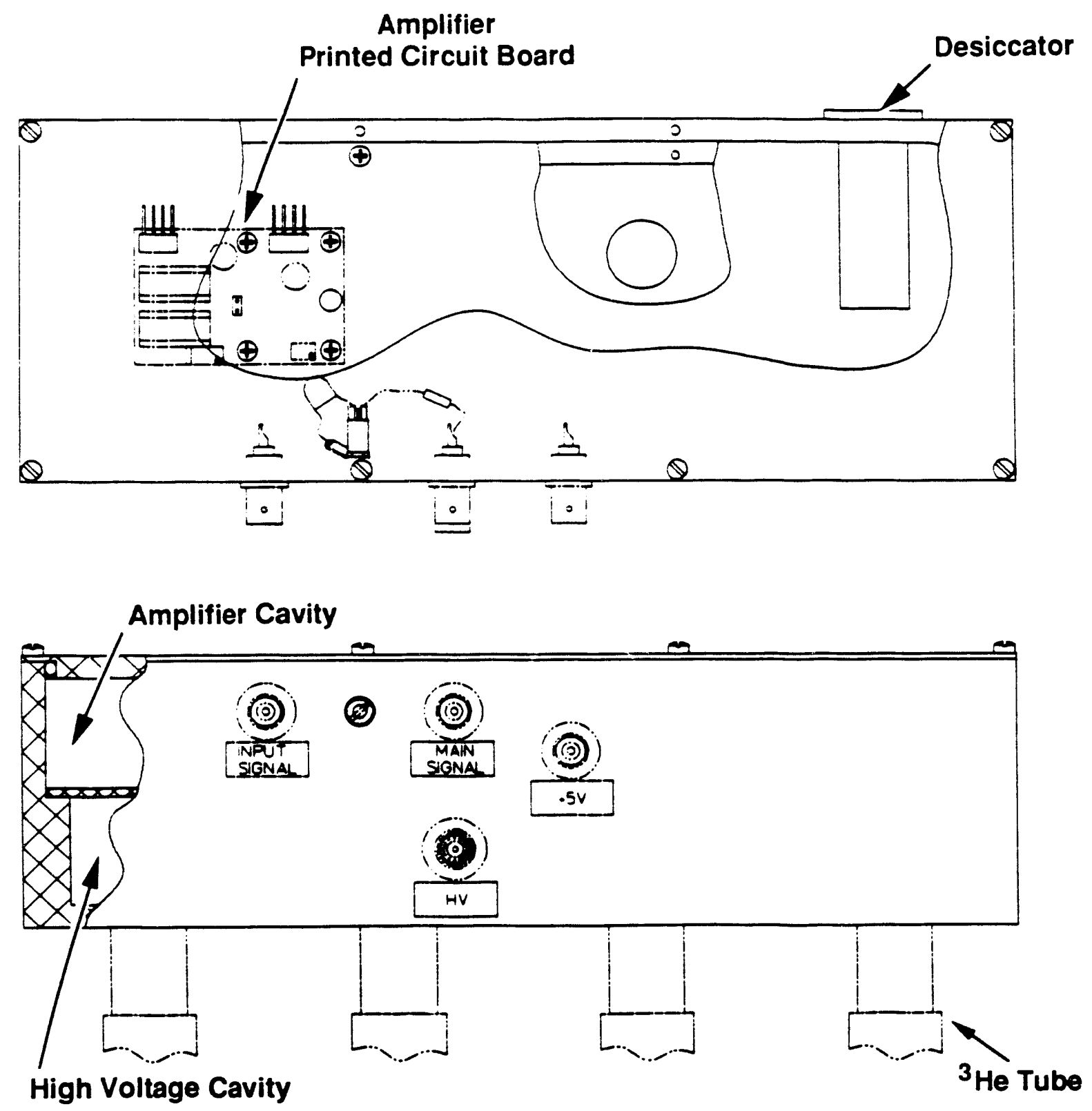

Fig. 1. Pass-through shuffler junction bax, conventional design. 

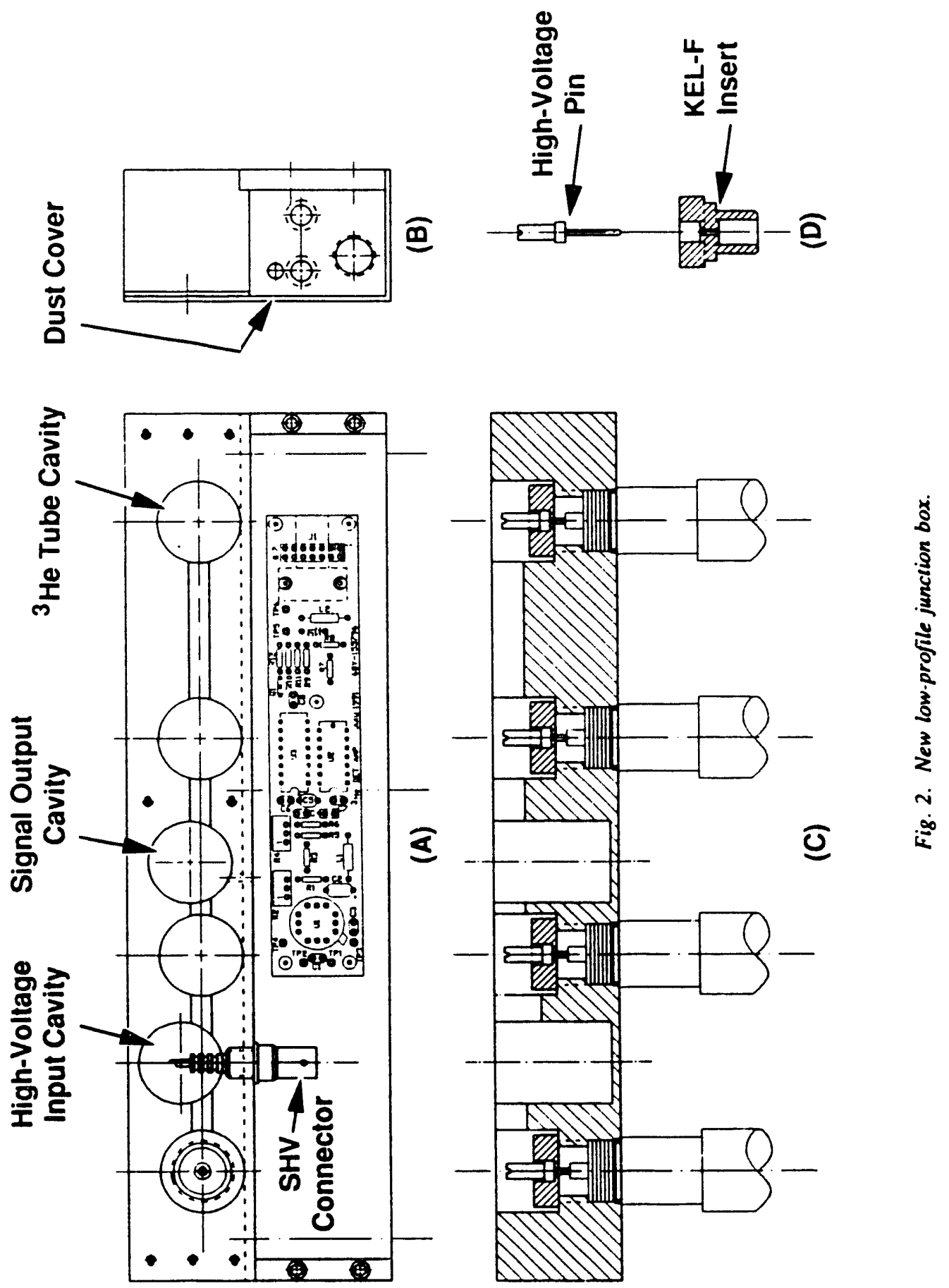


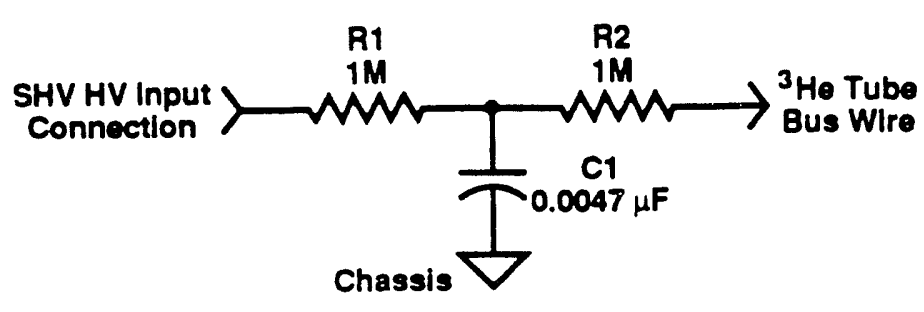

(A) HIGH VOLTAGE INPUT FILTER

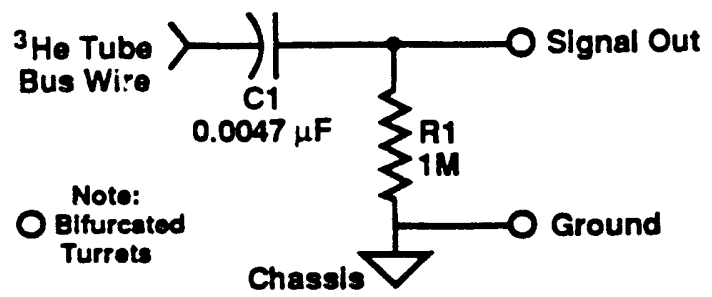

(B) SIGNAL OUTPUT CIRCUIT

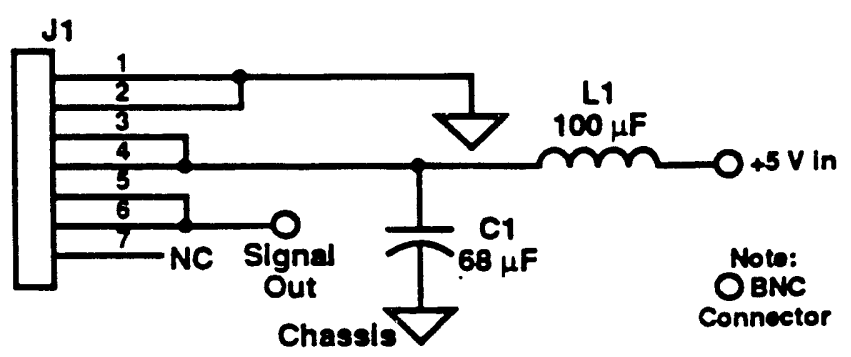

(C) CONNECTOR CARD SCHEMATIC

Fig. 3. Low-profile junction box circuit boards.

coupling capacitor is a ceramic disk made by TDK Corporation of America and was selected to withstand the high voltage and have a low-corona discharge, which can elicit superfluous counts from the detector.

After soldering the bus wire on the pins and installing the input/output printed-circuit cards, we filled the six cavities of the junction box with a potting material to seal moisture from the high-voltage components. An epoxy resin, STYCAST 2651 made by Emerson and Cuming, Inc., is used for the potting material. It was selected because it could be poured at room temperature and it had good high-voltage characteristics such as a dielectric strength of $450 \mathrm{~V} / 0.001$ in. and volume resistivity of $5 \times 10^{16} \Omega-\mathrm{cm}$. O-rings are used at the base of the ${ }^{3} \mathrm{He}$ tubes and the junction box to keep moisture from getting into the area where the wbes interface with the junction box.

Figure 2, par (B), shows a cross section from the end view of the junction box. This view shows the potted cavity cover and the dust cover of the junction box. The dust cover is a one-piece, right-angle cover, which fastens to the main junction box body with two screws on the top for easy removal of the cover. A separate cavity cover is used to contain the magnetic field in the area of the high-voltage components while adjustments are made on the amplifier card. This view also shows the connector interface panel; there is one for each bank of three junction boxes. Three cables are connected to each bank: the high-voltage cable, the $+5 \mathrm{~V}$ power cable, and the output signal cable. Within the junction boxes, the high voltage is distributed to each junction box input cavity with coax cables and T-connectors. The $+5 \mathrm{~V}$ and the detector output signals are distributed between amplifier cards using a sevenconnector flat cable. A circuit diagram of the connector printed-circuit card is shown in Fig. 3, part (C).

The drawing in Fig. 4 illustrates a typical detector bank consisting of three modules, each with four ${ }^{3} \mathrm{He}$ ubes and one amplifier board. This figure also shows how the amplifiers in the three modules are connected by flat cables. The amplifier for the conventional junction box was repackaged to provide a more narrow printed-circuit card that better fits the new junction box layout. A schematic of the amplifier is shown in Fig. 5. The analog amplifier circuit and digital circuit are identical to the conventional junction box amplifier. The analog amplifier is a hybrid, charge-sensitive preamplifier discriminator made by Amptek, Inc. that has proven very reliable and is well suited for this application. Digital processing includes a single-shot that produces a 50-ns output pulse for each neutron event, a line driver, and a circuit to $O R$ the signals from ocher amplifier cards. Light emitting diodes (LEDs) are used with the detectors to indicate visually when neutron events are being processed and are used during adjustment of the amplifier input threshold. In order to view the light from the LEDs after the detector module is assembled, a plastic fiber-optic cable conducts the LED light to a display panel at the electronics rack. The fiber optic interface to the LED uses a housing and fiber connectors made by AMP.

The new junction box was extensively tested to verify its performance and to determine if the potting material is successful in prohibiting moisture from entering the high-voltage area of the junction box. For comparison, identical tests were performed side-by-side with the conventional junction box because its performance is well established. Therefore, test data are presented for both junction boxes. Figure 6 shows the high-voltage-profile calibration plots for the junction boxes. The profile was taken using an AmLi source, the ${ }^{3} \mathrm{He}$ tubes were inserted in polyethylene, and the data were taken with a JSR-11 coincidence counter. As the plots indicate, the highvoltage profiles are almost identical.

Results of humidity tests on the new junction box before the potting material was added are shown in Fig. 7. The effects of humidity began to show at about $40 \%$ relative humidity. Above that amount, the counts of the unpotted new junction box increased considerably as shown in the graph. Increased humidity did not affect the counts for these tests on the conventional junction box because the desiccators were still effective in removing the small amount of moisture entering the junction box. The humidity tests were performed using an environmental chamber that can control both temperature and relative humidity. The temperature was set $1080^{\circ} \mathrm{F}$ for the unpotted humidity tests.

The moisture resistance of the poting material was tested by placing the potted junction box in the environmental 


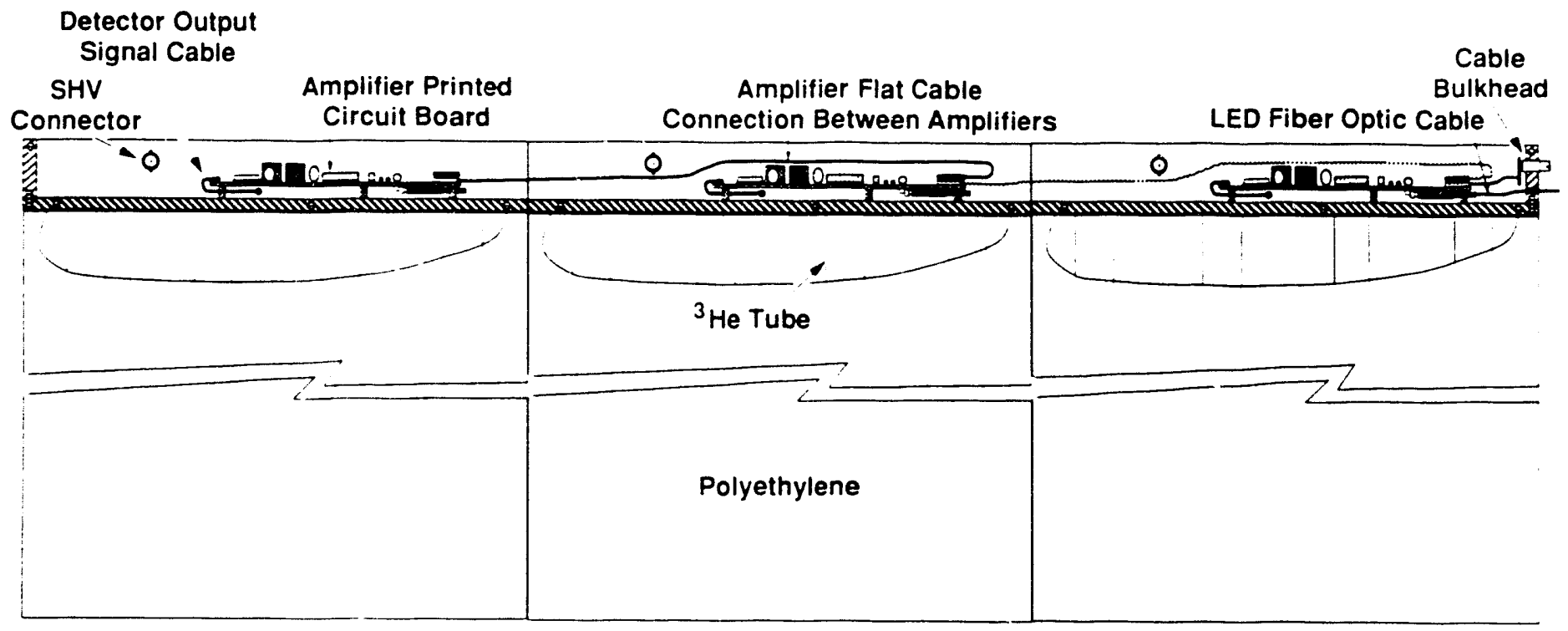

Fig. 4. Neutron detector bank.

chamber with high humidity at several different temperatures for a long time and measuring the background count rate. Tests were performed on the conventional junction box at the same time for comparison. Background tests were done with the ${ }^{3} \mathrm{He}$ tubes covered with sheets of cadmium but without using polyethylene. JSR-11 coincidence counters were used to collect the data and the results are shown in Fig. 8. The count rate shown is the counter's total counts accumulated over 1000 seconds and averaged over many hours, that is, 24 hours in many of the tests. The standard deviation of the count rates for the tests, which contained up to one hundred 1000 -second runs ranged from 0.001 to 0.006 counts/s. The high voltage was set at $1680 \mathrm{~V}$ and the gate length of the JSR-11 was set to $128 \mu \mathrm{s}$. The background count rate for the new low-profile junction box shows a slight increase over the 40 days of testing. The same upward trend was also present in the conven- tional junction box. After about 30 days of continued exposure to the $90 \%$ relative humidity and the temperature of from $90^{\circ} \mathrm{F}$ to $120^{\circ} \mathrm{F}$, the conventional junction box experienced some high-count rates for a couple of days, but then the count rates returned to normal. The desiccators had turned slightly pink by this time, indicating they were becoming saturated with moisture. Time did not permit testing beyond the 40 days. We believe that this was sufficient time at elevated humidity and temperatures to demonstrate the moisture resistance capability of the potting material.

In conclusion, we designed and tested a simple junction box that provides counting performance equal to that of the proven junction box design and has several significant improvements. This new junction box is cheaper to make, its design allows easy removal and replacement of ${ }^{3} \mathrm{He}$ tubes, and it is expected to require less maintenance. 


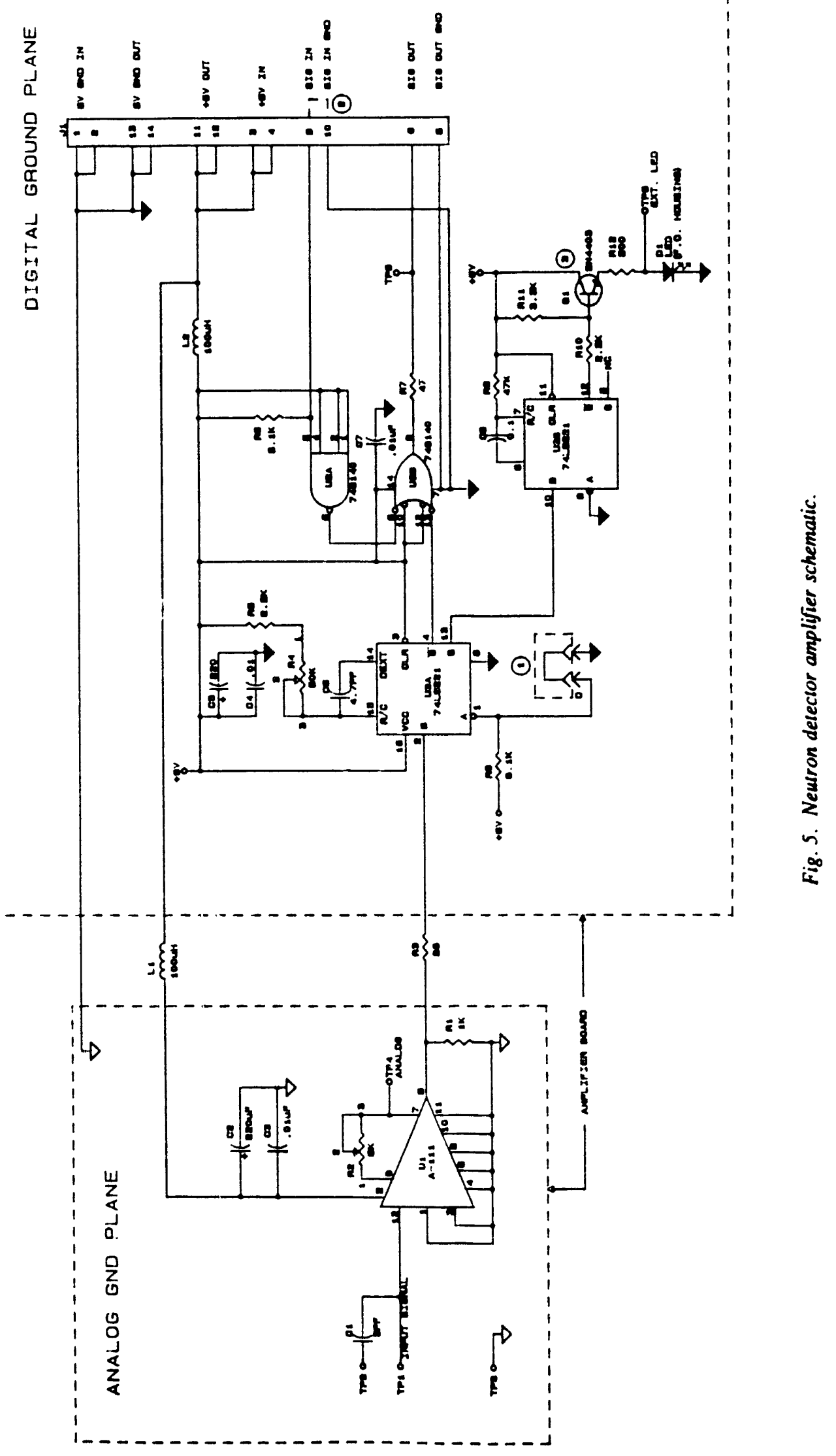




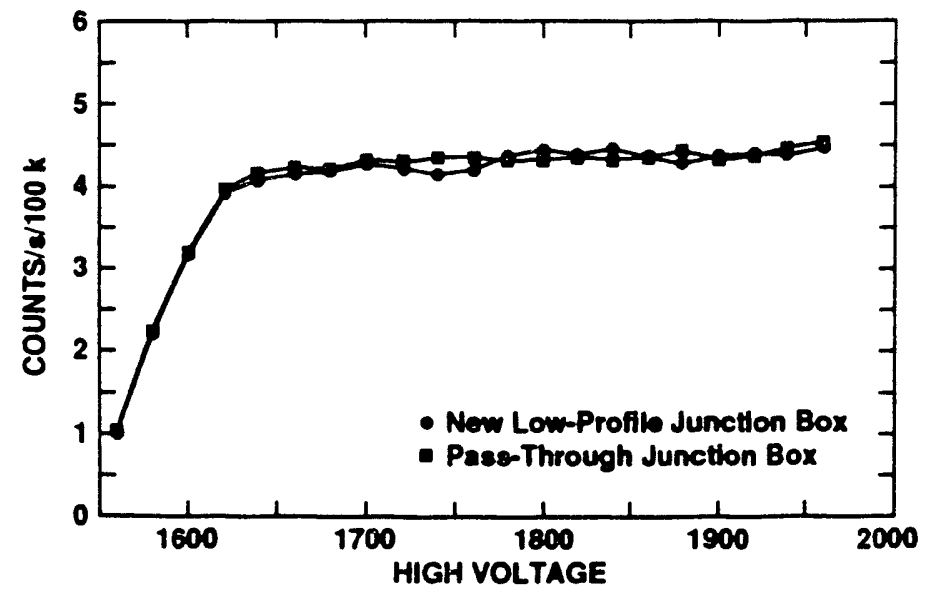

Fig. 6. High-voltage-profile calibration.

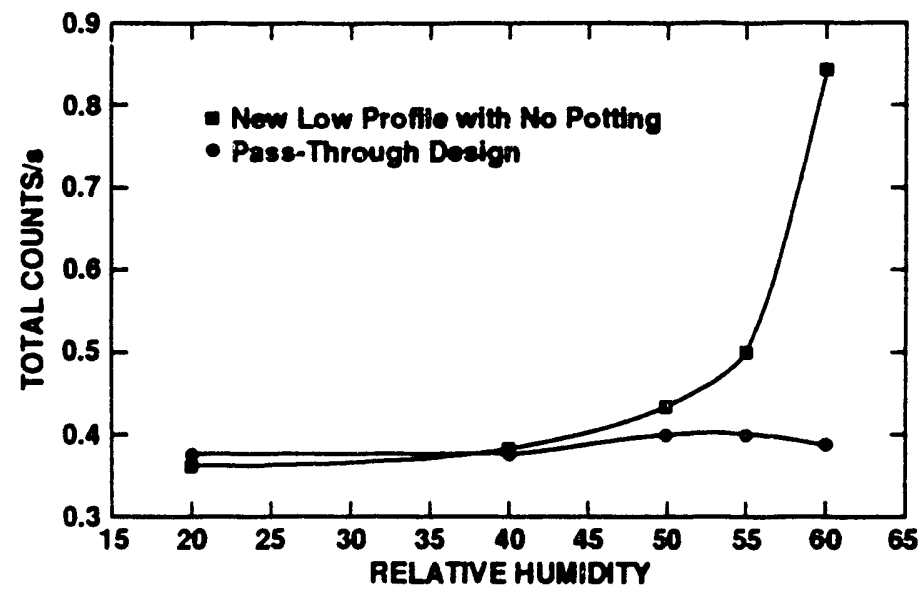

Fig. 7. Humidity test results on unpotted junction box.

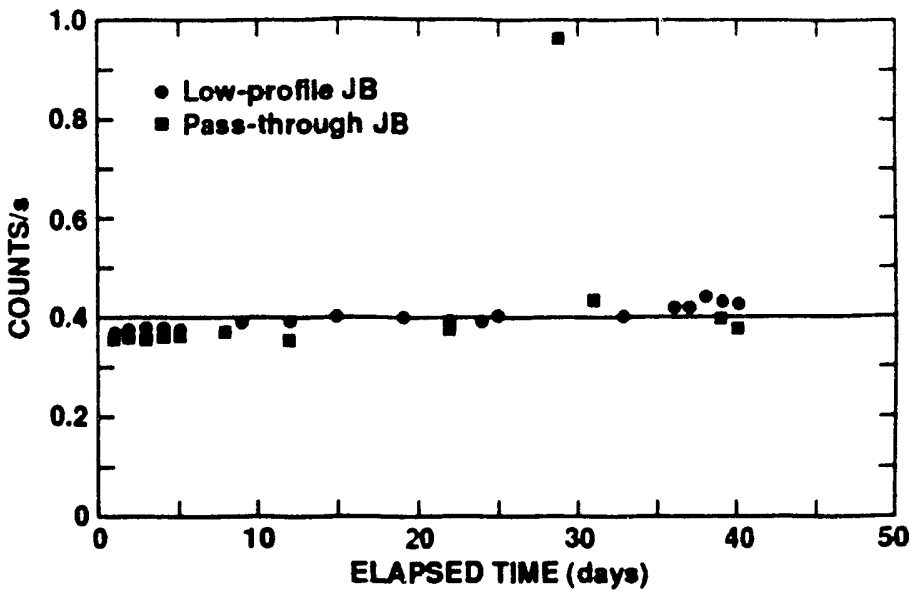

Fig. 8. Long-term humidity test results. 

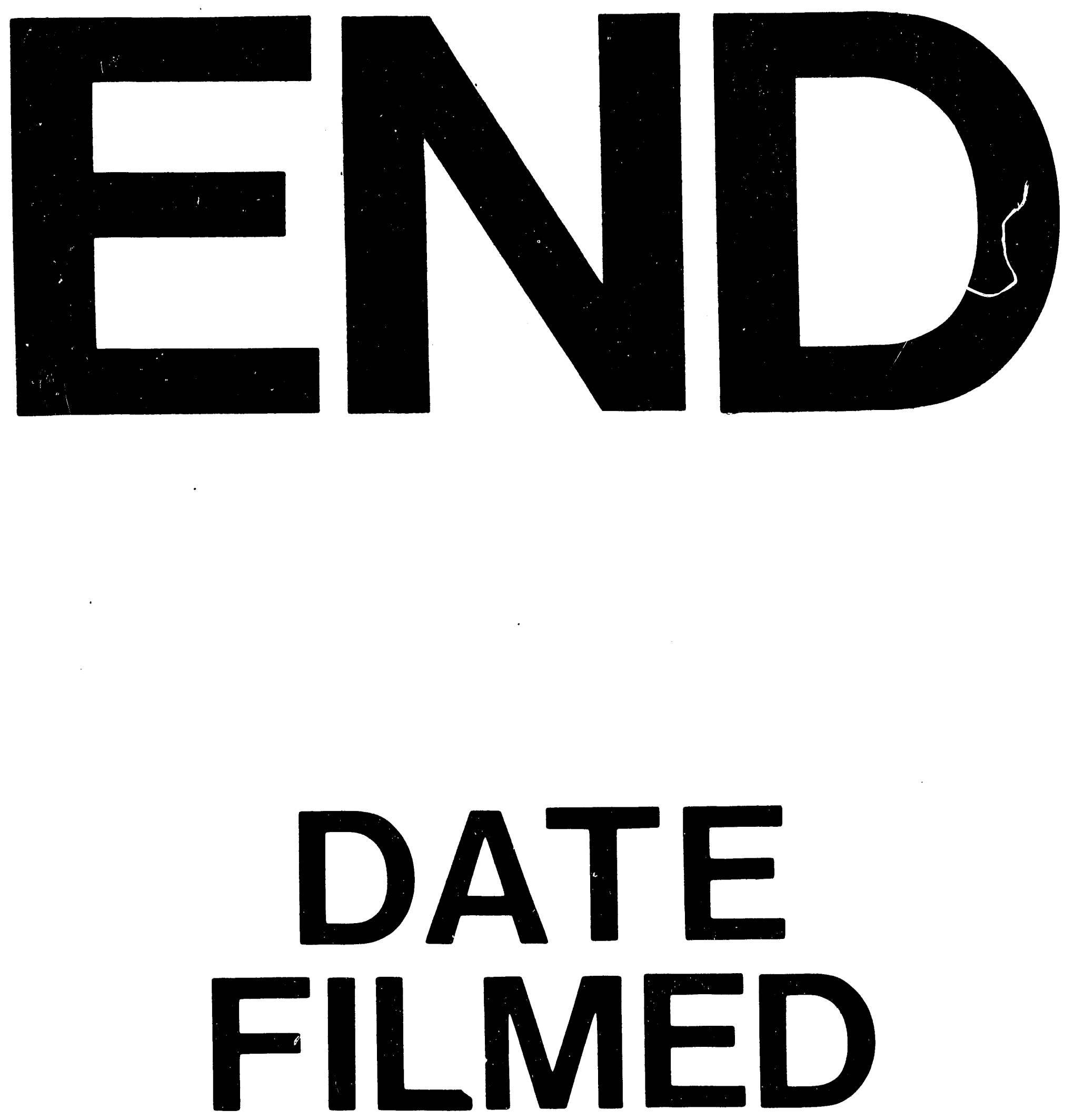

1

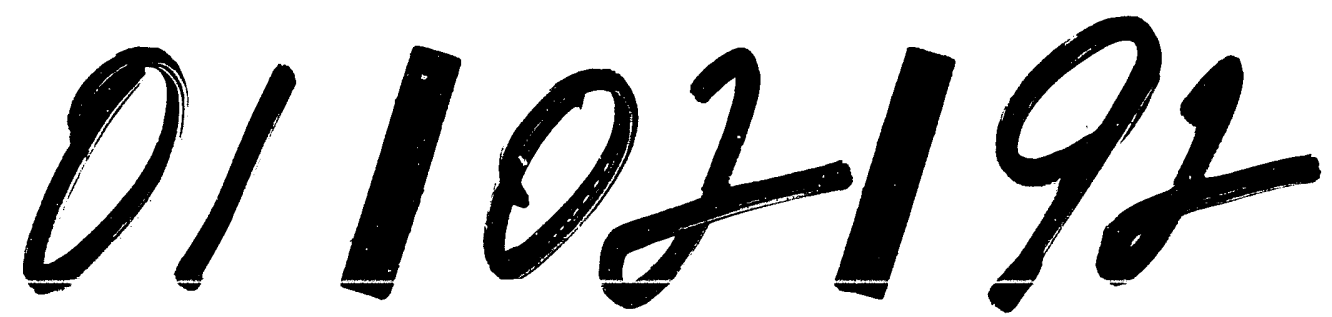


